\title{
RUPTURA BILATERAL DO QUADRÍCEPS NA DOENÇA RENAL CRÔNICA
}

\section{BILATERAL QUADRICEPS RUPTURE IN CHRONIC KIDNEY DISEASE}

DOI: $10.5380 /$ rmu.v1i4.40695

Gabriel Canto Tomazini ${ }^{1}$, Lucas Daniel Carstens ${ }^{1}$, Edmar Stieven Filho ${ }^{1}$ Marco Aurélio Taucci de Castro Júnior ${ }^{2}$.

\section{RESUMO}

A ruptura bilateral do tendão do quadríceps é uma lesão rara, mas pode ser encontrada em pacientes com doença renal crônica após traumas mínimos. Este relato apresenta um caso de ruptura bilateral do tendão do quadríceps de uma mulher de 32 anos de idade, portadora de doença renal crônica após queda de mesmo nível. A correção cirúrgica foi realizada com âncoras fixadas ao tendão e suturadas pela técnica de Krackow. No pós-operatório, a reabilitação foi realizada com fisioterapia e ao longo da evolução adquiriu satisfatória amplitude de movimento ativa.

Palavras-Chave: Ruptura, quadríceps, hiperparatireoidismo, falência renal crônica.

\section{ABSTRACT}

Bilateral rupture of the quadriceps tendon is a rare injury, but may be found in patients with chronic kidney disease after minimal trauma. This report presents a case of bilateral rupture of the quadriceps tendon in a 32-year-old woman with chronic kidney disease after a low level falling. Surgical correction was performed with anchors fixed to the tendon and sutured by Krackow technique. Postoperatively, the rehabilitation was done with physiotherapy and throughout evolution acquired satisfactory active range of motion.

Key-Words: rupture, quadriceps, hyperparathyroidism, chronic kidney failure 


\section{REVISTA MÉDICA DA UFPR}

\section{INTRODUÇÃO}

A disfunção renal persistente, geralmente secundária a uma patologia lentamente progressiva pode levar a um estado de doença renal crônica (DRC). $\mathrm{Na} \mathrm{DRC}$ a regeneração do parênquima renal não ocorre e, por isso, a perda da unidade estrutural básica do rim é irreversível. ${ }^{1}$ A hemodiálise é um tratamento que permite uma maior expectativa e melhor qualidade de vida para o portador de DRC, entretanto a incidência de complicações é relevante ${ }^{2}$.

Uma das complicações é o hiperparatireoidismo secundário, que pode levar a alterações no balanço do cálcio e fosfato, com a deposição de complexos minerais em estruturas extra ósseas, peri articulares e perivasculares. Esse processo gera uma fragilidade do aparelho locomotor que pode se manifestar através de fraturas e rupturas tendinosas por pequenos traumas ${ }^{3}$.

A ruptura do tendão do quadríceps é incomum e ocorre principalmente em idosos ou em traumas graves. As rupturas bilaterais são raras e estão relacionadas a doenças sistêmicas ${ }^{4}$. Na literatura já foram descritos casos relacionando a ruptura do tendão do quadríceps às doenças crônicas, tais como DRC, artrite reumatoide, lúpus eritematoso sistêmico, arteriosclerose, diabetes mellitus, hiperparatireoidismo primário e secundário, gota, tuberculose e vasculites. Neste trabalho vamos relatar um caso relacionado à $\mathrm{DRC}^{5}$.

\section{RELATO DO CASO}

Paciente feminina, 32 anos de idade, procurou atendimento após queda de mesmo nível com apoio sobre os joelhos. Na admissão apresentava dor em região supra patelar direita e esquerda e limitação nos movimentos de extensão e flexão das pernas, com deambulação prejudicada.

O exame físico demonstrava um intervalo palpável supra patelar tanto a direita quanto a esquerda. Exames de imagem foram realizados (radiografia e ultrassonografia), e evidenciaram a ruptura do tendão do músculo quadríceps em ambos os membros. (FIGURAS 1 E 2).

A história clínica incluía insuficiência renal crônica por atrofia renal e tratamento com hemodiálise iniciado aos 17 anos. Um ano e meio após o início da terapêutica foi realizado transplante renal (FIGURA 3), o qual permaneceu efetivo por seis anos, evoluindo com nefropatia crônica do enxerto. $\mathrm{Na}$ época do trauma realizava hemodiálise através de fístula arteriovenosa femoral direita.

Os exames laboratoriais indicavam anemia (dosagem de hemoglobina 10,6g/dL; hematócrito $30,9 \%$ ) e um hiperparatireoidismo secundário à insuficiência renal crônica (creatinina 11,07mg/dL; PTH $1.952 \mathrm{pg} / \mathrm{ml}$; cálcio $8,1 \mathrm{mg} / \mathrm{dL}$; fosfato $5,97 \mathrm{mg} / \mathrm{dL}$ ).

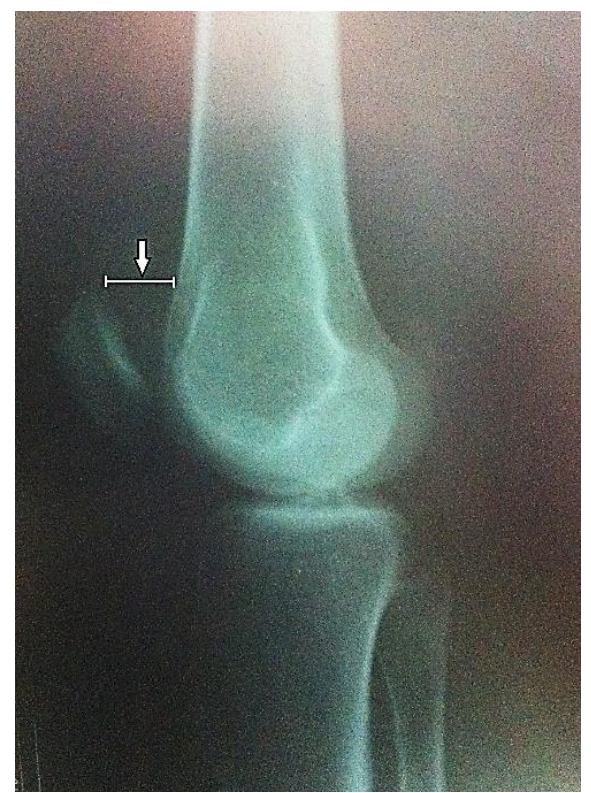

FIGURA 1:Radiografia pré-operatória do joelho direito: presença de inclinação anterior do pólo superior da patela indicada pela seta branca.

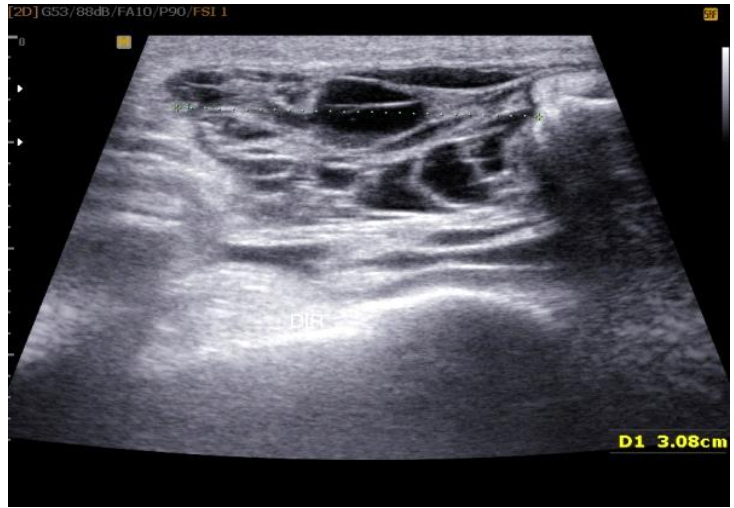

FIGURA 2: Ultrassonografia do tendão quadriciptal direito: presença de retração superior com líquidos multisseptados evidenciando ruptura.

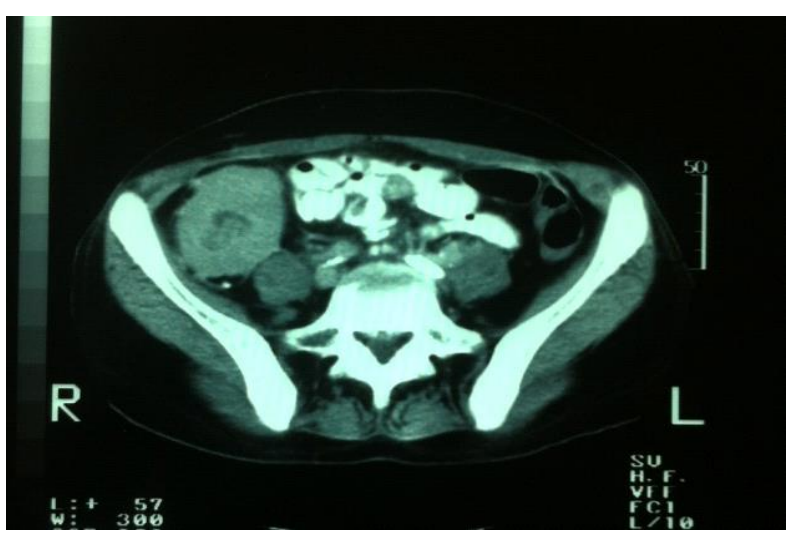

FIGURA 3: Tomografia computadorizada evidenciando rim transplantado em fossa ilíaca direita quando este ainda era efetivo 
As rupturas dos tendões quadricipitais foram abordadas por acessos anteriores longitudinais com incisões medianas de dez centímetros em região anterior dos joelhos. Após a exploração foi identificada área de desinserção dos tendões na região superior proximal da patela além de lesões retinaculares, em seguida feito a retirada de hematoma residual e debridamento da lesão. (FIGURA 4).

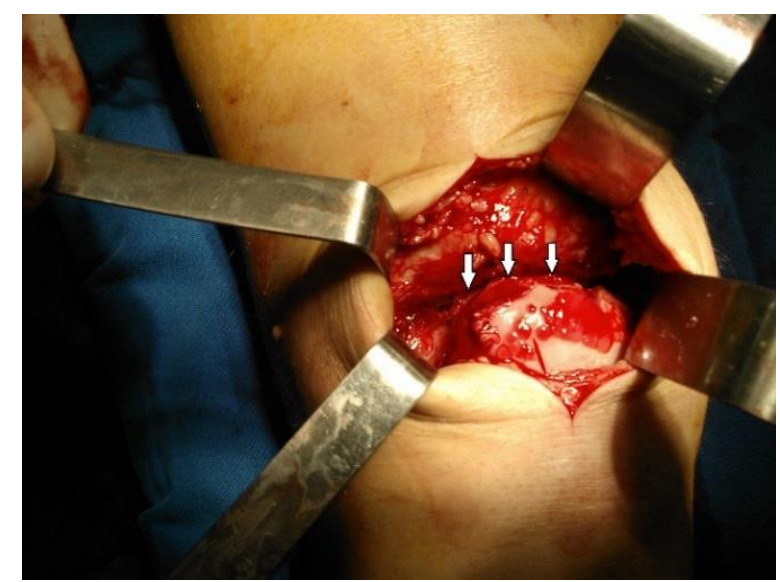

FIGURA 4: Aparência do tendão quadricipital direito rompido no pólo superior da patela no intraoperatório. Setas brancas identificam a desinserção.

A reinserção tendinosa à esquerda foi feita com o uso de duas âncoras no polo superior da patela, uma lateral e outra medial a linha mediana da patela. Essas âncoras foram fixadas ao tendão através de fios de poliéster inabsorvíveis número cinco (Ethibond ${ }^{\circledR}$ ) e suturadas pela técnica de Krackow, com o joelho fletido a 60 graus.

À direita, a ancoragem não foi feita devido à baixa qualidade óssea. A reinserção foi feita com pontos transversos atravessando a patela com o uso de fios agulhados inabsorvíveis número cinco (Ethibond ${ }^{\circledR}$ ) e sutura do tendão também com a técnica de Krackow, com o joelho em flexão de 60 graus.

No pós-operatório, os joelhos foram mantidos em extensão através de órteses por duas semanas. A reabilitação foi feita com fisioterapia inicialmente passiva e ao longo da evolução adquiriu satisfatória amplitude de movimento ativa. A radiografia de dois meses após o procedimento cirúrgico demonstrava patela esquerda sem inclinação anterior, com as duas âncoras e deposição de cálcio na região da cicatrização da ruptura. (FIGURAS 5 E 6).

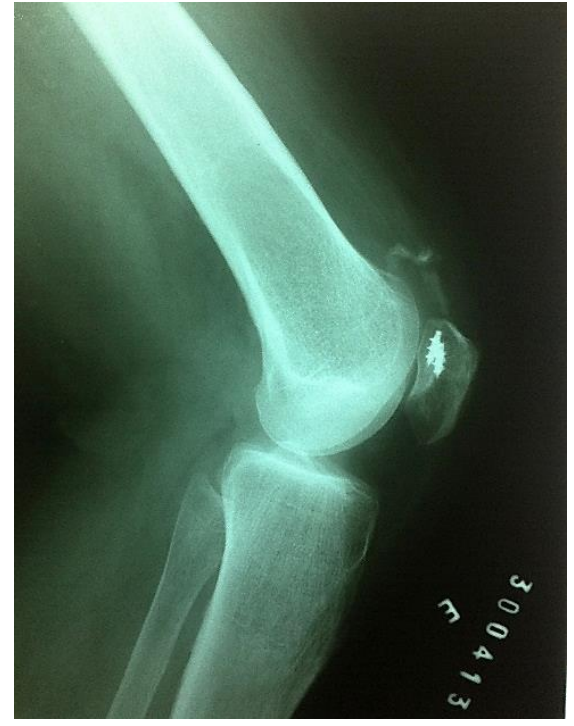

FIGURA 5: Raio-x de joelho esquerdo semi-fletido com 2 meses de pós-operatório demonstrando patela anatômica com duas âncoras em seu polo superior.

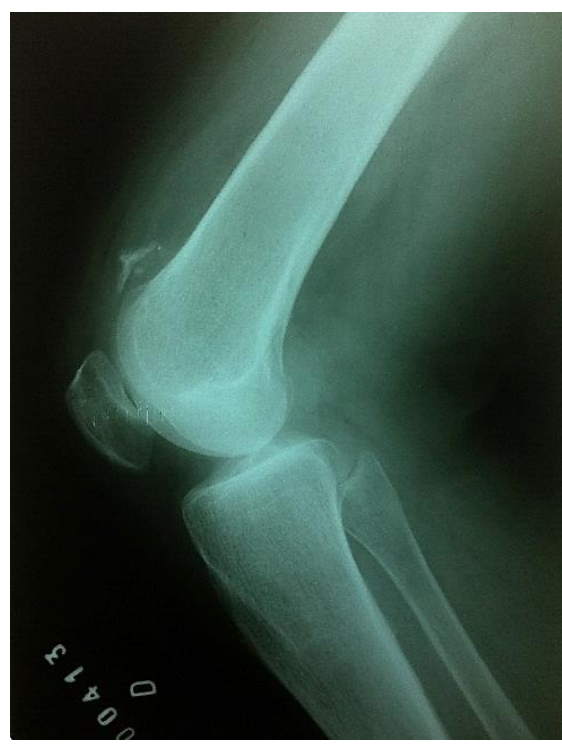

FIGURA 6: Raio-x de joelho direito semi-fletido com 2 meses de pós-operatório demonstrando patela anatômica.

\section{DISCUSSÃO}

A ruptura bilateral do tendão do quadríceps é uma complicação rara, e quando ocorre geralmente está relacionada à doenças sistêmicas crônicas, como a DRC avançada. A duração da falência renal e o tempo em diálise estão intimamente associados a esse tipo de lesão ${ }^{6}$. O principal mecanismo de trauma, nesses casos, é a contração súbita e excêntrica do músculo quadríceps com flexão do joelho concomitante aos pés fixos no solo?.

Há uma série de fatores que contribuem para a diminuição da elasticidade e resistência do tendão. Entre eles, a diminuição do aporte sanguíneo local, 
distúrbios do metabolismo de colágeno, micro traumas de repetição e calcificações tendinosas. Não há um consenso na literatura sobre essas causas ${ }^{8}$.

É mais comum a ruptura na junção osteoligamentar, devido à menor perfusão e, especificamente, em casos de DRC com elevados índices de PTH, deposição de fosfato de cálcio nesta região. Neste caso, temos uma paciente com DRC em diálise há sete anos com hiperparatireoidismo secundário, que possui papel relevante na fisiopatologia $^{9}$ e que sofreu um trauma com mecanismo compatível com a lesão.

O diagnóstico pode ser feito por meio da história clínica, do exame físico e de exames de imagem. História de trauma simples envolvendo rápida e excêntrica contração muscular com o pé apoiado e o joelho parcialmente fletido, ou espontaneamente em pacientes com doenças sistêmicas, podem sugerir um quadro de ruptura quando o exame físico apresenta dor, edema e incapacidade funcional com perda da extensão ativa do joelho, mas com flexão intacta.

Neste relato, a história e o exame físico compatíveis com a ruptura, associados a exames de raio-x e ultrassonografia estabeleceram o diagnóstico. Em casos de dúvida diagnóstica e de lesões associadas, poderia se lançar mão da ressonância nuclear magnética (RNM) e tomografia computadorizada.

Deve-se ter em mente que um paciente em diálise está susceptível à lesões osteomusculares, principalmente se jovem e ativo. Portanto o médico assistente deve estar atento à história e sintomas sugestivos. Uma vez que a maioria dos autores concorda que o diagnostico rápido, a intervenção cirúrgica adequada e a fisioterapia precoce são imprescindíveis para o sucesso da recuperação anatômica e funcional ${ }^{10}$.

\section{REFERÊNCIAS}

1- FAUCl, A. S., BRAUNWALD, E., KASPER, D. L., HAUSER, S. L., LONGO, D. L., JAMESON, J. L., LOSCALZO, J. Harrison's Principles of Internal Medicine, 17a ed, McGraw Hill Medical, 2008.

2- Kim, Y.H., SHAFI, M., LEE, Y.S., KIM, J.Y., KIM, W.Y., HAN, C.W. Spontaneous and simultaneous rupture of both quadriceps tendons in a patient with chronic renal failure. A case studied by MRI both preoperatively and postoperatively. Knee Surg Sports Traumatol Arthrosc. 2006;14:55-9.

3- MATOKOVIC, D., MATIJACEVIC, B., PETRIC, P., CRNKOVIC, T., SKORVAGA, S. A case report of spontaneous concurrent bilateral rupture of the quadriceps tendons in a patient with chronic renal failure. Ther Apher Dial. 2010;14:104-7.
4- SHAH, M., K. Simultaneous bilateral quadriceps tendon rupture in renal patients. Clin Nephrol. 2002;58:118-21.

5- RAJEEV, A., SENEVIRATHNA, S., RADHA, S., KASHAYAP, N., S. Bilateral simultaneous rupture of the quadriceps tendon in a patient with psoriasis: a case report and review of the literature. J Med Case Reports. 2011; 5: 331.

6- RIBBANS, W.,J., ANGUS, P., D. Simultaneous bilateral rupture of the quadriceps tendon. $\mathrm{Br} J$ Clin Pract. 1989;43:122-5.

7- NEUBAUER, T., WAGNER, M., POTSCHKA, T., RIEDL, $M$. Bilateral, simultaneous rupture of the quadriceps tendon: a diagnostic pitfall? Report of three cases and meta-analysis of the literature. Knee Surg Sports Traumatol Arthrosc 2007;15:43-53.

8- PETERSEN, W., STEIN, V., TILLMAN, B. Blood supply of the quadriceps tendon. Unfallchirurg1999;102:5437 .

9- KARA, A., SARI, S., SEKER, A., OZTURK, I. Osteotendinous repair of bilateral spontaneous quadriceps tendon ruptures with the Krackow technique in two patients with chronic renal failure.Acta Orthop Traumatol Turc 2013;47(1):68-71.

10- CHEN, C., CHU, P., HUANG, G., WANG, S., WU, S. Spontaneous Rupture of the Patellar and Contralateral Quadriceps Tendons Associated with Secondary Hyperparathyroidism in a Patient Receiving Long-term Dialysis. J formos Med Assoc. 2006, 105: 941-5. 